Índice mensual de incertidumbre financiera y económica (IMIFE) para la economía colombiana

Juan Manuel Candelo-Viáfara 
Juan Manuel Candelo-Viáfara

Índice mensual de incertidumbre financiera y económica (IMIFE) para la economía colombiana

Resumen: Este articulo desarrolla un indice que permite medir los niveles de incertidumbre en la economía colombiana, al cual se le ha nombrado indice mensual de incertidumbre financiera $y$ económica (IMIFE). Se utiliza la metodología de factores dinámicos, usando los históricos de los precios internacionales del petróleo, carbón, gas y la tasa representativa del mercado peso por dólar (TRM). Como resultados relevantes, se encuentra que el indice aumentó en momentos de crisis económica, como lo fueron los años 2008 (crisis subprime) y 2020 (Covid-19). Igualmente, se identificó un comportamiento inverso entre el valor de las acciones de las empresas colombianas y el IMIFE, además de un impacto negativo del nivel de incertidumbre sobre la economia nacional. Este indice puede ser utilizado, tanto por los elaboradores de política económica, los inversores del mercado accionario y los gerentes de las organizaciones.

Palabras clave: incertidumbre, indice, economía colombiana, factores dinámicos, materias primas energéticas, tasa de cambio.

Clasificación JEL: C22, C43, D80, D81, Q02.

\section{Monthly Financial and Economic Uncertainty Index (IMIFE) for the Colombian Economy}

Abstract: This document develops an index that allows measuring the levels of uncertainty in the Colombian economy, which has been given the name of the IMIFE monthly index of financial and economic uncertainty. For this, the dynamic factors methodology was used using the historical ones of the international prices of oil, coal, gas, and the representative market rate of the peso per dollar (TRM). As relevant results, it is found that the index increased in times of economic crisis such as in 2008 (crisis suppressed) and 2020 (Covid-19). Likenise, an inverse behavior was identified between the value of the shares of Colombian companies and IMIFE; in addition to a negative impact of this index on the national economy. This index can be used by both economic policy makers, stock market investors, and organization managers.

Keywords: Uncertainty, index, Colombian economy, dynamic factors, energy raw materials, exchange rate.

\section{(cc) BY-NC-SA}

Este artículo y sus anexos se distribuyen por la revista Lecturas de Economía bajo los términos de la Licencia Creative Commons Atribución-NoComercial-CompartirIgual 4.0. https://creativecommons.org/licenses/by-nc-sa/4.0/ 


\section{Un indice mensuel d'incertitude financière et économique (IMIFE) pour l'économie colombienne}

Résumé: Cet article propose un indice qui mesure les niveaux d'incertitude dans l'économie colombienne, appelé « indice mensuel d'incertitude financière et économique, IMIFE ». Nous adoptons la méthodologie des facteurs dynamiques en prenant les prix internationaux historiques du pétrole, du charbon, du gaz et le taux de change peso/dollar (TRM). Nous constatons que l'indice augmente dans les périodes de crises économiques, comme par exemple, en 2008 (la crise supprime) et en 2020 (la crise du Covid-19). Nous avons également identifié un comportement inverse entre la valeur des actions des entreprises colombiennes et l'IMIFE, en plus d'un impact négatif du niveau d'incertitude sur l'économie nationale. Cet indice peut être utilisé à la fois par les décideurs politiques, les investisseurs boursiers et les gestionnaires des entreprises.

Mots clés: Incertitude, indice, économie colombienne, facteurs dynamiques, matières premières énergétiques, taux de change.

Cómo citar / How to cite this item:

Candelo-Viáfara, J. M. (2021). Índice mensual de incertidumbre financiera y económica (IMIFE) para la economía colombiana. Lecturas de Economía, (95), 85-104.

https://doi.org/10.17533/udea.le.n95a343318 


\title{
Índice mensual de incertidumbre financiera y económica (IMIFE) para la economía colombiana
}

\author{
Juan Manuel Candelo-Viáfara $\odot^{a}$ \\ -Introducción. -I. Revisión de literatura. -II. Modelo econométrico. -III. Discusión de \\ resultados. -Conclusiones. -Agradecimientos. -Referencias.
}

Primera versión recibida el 27 de julio de 2020; versión final aceptada el 08 de febrero de 2021

\section{Introducción}

El análisis de la incertidumbre cobra importancia en la medida que la misma la definen autores como Knight (1921) y Keynes (1921; 1939) como el desconocimiento de los eventos futuros, lo que repercute en la toma de decisiones de los agentes a la hora de tomar decisiones. Al analizar la incertidumbre, diferentes autores han mencionado elementos que muestran sus impactos en el bienestar económico, como lo es el aumento del costo de oportunidad a la hora de invertir (Bernanke, 1983), poca creencia en las instituciones (Streb, 2000), contracción en el consumo, empleo y el nivel de producción (Bloom, 2009; Cerda et al., 2016 y Haddow et al., 2013).

De igual forma, la economía colombiana se ha caracterizado por su relación con la producción de materias primas energéticas como lo son el petróleo, el carbón y el gas, encontrando así una relación positiva entre los precios de estos bienes y la actividad económica nacional (Gómez, 2015; González \& Hernández, 2016; Mendoza \& Vera, 2010 y Poncela et al., 2017). En esa línea, hay que resaltar que Basher y Sadorsky (2016), Brahmasrene et al. (2014) y de Truchis y Keddad (2016), han identificado que la alta volatilidad de las materias primas están asociadas con momentos de incertidumbre, permitiendo así inferir que, dado que la economía colombiana relaciona su crecimiento con estas materias primas, es factible desarrollar un índice que

a Juan Manuel Candelo-Viáfara: profesor hora cátedra. Universidad del Valle, Colombia.

Dirección electrónica: juan.candelo@correounivalle.edu.co

https://orcid.org/0000-0002-4152-5597 
permita medir la incertidumbre basado en los precios de las materias primas energéticas.

Dado lo anterior, este artículo se propone como objetivo desarrollar un índice que permita medir los niveles de incertidumbre en la economía colombiana. Para ello, se utilizó la metodología de factores dinámicos propuesto por Geweke (1977) usando los datos históricos de los precios del barril de petróleo WTI y Brent, los precios del carbón australiano, el precio del gas y la tasa representativa del mercado (TRM). Seguido de esta introducción, se encuentran las secciones revisión de literatura, modelo econométrico, discusión de resultados y conclusiones.

\section{Revisión de literatura}

El no conocer qué eventos se aproximan, hace referencia a un término llamado incertidumbre (Knight, 1921; Keynes, 1921; 1939). En esta línea diversos autores como Bernstein (1998) afirman que la incertidumbre se refleja en distintas variables macroeconómicas. Por tanto, Ali et al. (2017), Cooper et al. (2004), Daniel y Moskowitz, (2016) y Gervais et al. (2001) aseveran que la incertidumbre se asocia al comportamiento del sistema económico. Igualmente, hay que anotar que la incertidumbre también se ve asociada al valor de las empresas en el tiempo (Asness et al., 2013 y Moskowintz et al., 2012). Así, diversos investigadores han invertido esfuerzos con el fin de entender las anomalías producidas por este elemento, como Frazzini, (2006), Johnson (2002) y Liu et al, (2008) quienes analizan la incertidumbre desde la racionalidad; a su vez, Cooper et al. (2004), Daniel et al. (1998) y Hong y Stein (1999) plantean el análisis de la incertidumbre desde el comportamiento conductual. Los resultados de dichas investigaciones mencionan que en los momentos de mayor incertidumbre hay información heterogénea que genera fricción en los mercados y características específicas de las empresas. En esa medida Cooper et al (2004) y Novy-Marx (2012) identifican que la historia de las variables macroeconómicas es de gran relevancia para identificar anomalías que desencadenan en incertidumbre.

En los países latinoamericanos - y especialmente en Colombia- la apertura económica y la intensificación de las actividades minero-energéticas 
ha permitido que las fluctuaciones de las variables exógenas a la economía generen un impacto en ella (Ortiz, 2016). Autores como Candelo (2018), Gómez (2015), González y Hernández (2016), Mendoza y Vera (2010), Poncela et al. (2017), Oviedo y Sierra (2019) y Oviedo y Candelo (2020) han generado evidencia de que la economía colombiana se ve afectada por las fluctuaciones de las materias primas y la tasa de cambio. De acuerdo con lo anterior, autores como Basher y Sadorsky (2016), Brahmasrene et al. (2014), de Truchis y Keddad (2016), Golub (1983), Krugman (1983) y Lof y Nyberg (2017) han encontrado una relación entre los cambios de la tasa representativa del mercado con los precios de las materias primas. Ahora bien, esto toma relevancia en cuanto al análisis de incertidumbre, puesto que Basher y Sadorsky (2016), Brahmasrene et al. (2014), Brander y Krugman (1983), de Truchis y Keddad (2016), Golub (1983) y Lof y Nyberg (2017) plantean que la tasa de cambio y las materias primas son proxys de las anomalías en los mercados mundiales.

Igualmente se deben mencionar las investigaciones para Colombia que realizan índices para la medición de la incertidumbre. Entre ellas está la realizada por Partow (1996), el cual usa la productividad marginal del capital como herramienta para medir la incertidumbre. Igualmente, Birchenall (1997), quien usa la variación de la tasa de cambio y la inflación. Por otro lado, Delgado y Rincón (2017) utilizan los instrumentos fiscales en un modelo GARCH. Posada (2010) usa la tasa de cambio real y la inflación en un modelo GARCH para medir la incertidumbre y —en investigaciones más recientesGil y Silva (2019) utilizan la metodología planteada por Baker, Bloom y Davis (2016), donde se identifican en los medios de comunicación la palabra de incertidumbre para calcular el índice.

\section{Modelo econométrico}

En este artículo se siguió la metodología que permite la estimación de los factores no observables, donde se aplican los modelos dinámicos factoriales propuestos por Geweke (1977) y —a su vez- los aplica en una extensión del problema Stock y Watson $(2002,2005,2011)$. Los modelos dinámicos factoriales se pueden escribir de manera estática por la ecuación 1: 
Candelo-Viáfara: Índice mensual de incertidumbre financiera y económica (IMIFE)...

$$
\boldsymbol{X}_{t}=\Lambda \boldsymbol{F}_{t}+\boldsymbol{e}_{t}
$$

Así $\boldsymbol{X}_{t}$ es un vector de $N \times 1$ dimensiones, que contiene las variables de la TRM, los precios del petróleo, WTI y Brent, los precios del gas y los precios del carbón. Dicho vector está en función de la suma de dos componentes no observables: un componente que contiene la matriz de información de todas las variables, $\boldsymbol{F}_{t}$, y un componente idiosincrático o ruido blanco, $\boldsymbol{e}_{t}$, propio de cada variable. De igual manera, $\boldsymbol{\Lambda}$ es una matriz compuesta por las cargas de los factores, de dimensión $N \times K$ y el término $\boldsymbol{\Lambda} \boldsymbol{F}_{t}$ se define como los componentes comunes de $\boldsymbol{X}_{t}$. De igual manera, el término de error $\boldsymbol{e}_{t}$ de dimensión $N \times 1$, se caracteriza por tener media cero y varianza constante. Este último puede presentar una poca correlación transversal, en la medida que captura el componente idiosincrático.

El componente idiosincrático de cada variable que está asociada al componente autorregresivo esta dado por:

$$
x_{t}=\sum_{i=1}^{p} A_{i} e_{t-1} .
$$

El número de periodos está dado por $t=1,2,3, \ldots, N$ y $e_{t}$, representa un proceso de ruido blanco con media cero y varianza constante. De igual manera $A_{i}$, es una matriz de $N \times N$, que se describe por $A_{i}=$ $q_{1} A_{i}+q_{2} A_{i-2}+\cdots+q_{p} A_{p-N}$, donde $i=1,2,3 \ldots, N$ y $p$, es el número de rezagos. Por último, la estimación del factor dinámico se realiza por máxima verosimilitud.

\section{A. Datos}

Los datos usados para la estimación del índice de incertidumbre son de frecuencia mensual, desde enero de 2000 hasta junio de $2020^{1}$. Entre estos se encuentran las variables de tasa de cambio peso-dólar, el precio del barril de petróleo W'TI, el precio del barril de petróleo Brent, el precio spot del gas natural Henry Hub y el precio del carbón australiano. La utilización de

\footnotetext{
1 Estos datos se obtuvieron de FRED (FRED, 2020).
} 
estos datos en el índice se debe a que Basher y Sadorsky (2016), Brahmasrene et al. (2014), Brander y Krugman (1983), de Truchis y Keddad (2016), Golub (1983) y Lof y Nyberg (2017) plantean que la tasa de cambio y los precios de las materias primas energéticas son proxys de la incertidumbre, —además de la relación existente entre la economía colombiana con estas variables- lo cual es evidenciado por autores como Candelo (2018), Gómez (2015), González y Hernández (2016), Mendoza y Vera (2010), Oviedo y Sierra (2019), Oviedo y Candelo (2020) y Poncela et al. (2017). En esa línea, estos datos se obtuvieron del Banco Federal St. Lois (FRED, 2020).

La Figura 1 muestra el comportamiento de las variables de estudio. Primeramente, se puede notar que los precios del petróleo WTI, Brent, Carbón y gas en la primera mitad de la década del 2000 hasta el 2008, tuvieron un comportamiento al alza. Por otro lado, la TRM — dada su correlación inversa con los precios de estas materias primas - se comporta de manera contrapuesta. En el momento de la crisis del 2008, el precio de estas materias primas bajó considerablemente, e igualmente la tasa de cambio tiene un comportamiento contrario. Posterior a la crisis subprime, a diferencia del carbón y el petróleo, el gas tuvo un cambio estructural y siguió otra senda de comportamiento. Por otro lado, la cotización del petróleo crece después del 2009, llegando a otro de sus picos en el 2012. Después de este periodo, hay que mencionar que el valor de este hidrocarburo cae en el 2014 debido a una baja demanda, una extensión de la oferta, un cambio del objetivo por parte de la OPEP y la revaluación del dólar estadounidense (World Bank, 2015). Igualmente, también se debe mencionar que, en el 2018, cuando China y Estados Unidos empiezan la guerra comercial, el precio del petróleo baja ya su vez- la tasa de cambio aumenta significativamente. Respecto a crisis más recientes, en enero de 2020 — donde empieza la expansión del COVID19la tasa de cambio aumenta hasta puntos históricos y lo inverso ocurre con la valoración del petróleo. Además de lo anteriormente mencionado, se debe decir que la valoración del carbón tiene un comportamiento similar a los precios del petróleo.

La Tabla 1 muestra el coeficiente de correlación entre las variables de estudio. Aquí se puede identificar la relación negativa entre los precios de las materias primas del petróleo, carbón y gas respecto a la tasa representativa del 
mercado, lo cual se puede evidenciar, en la Figura 1 dado su comportamiento inverso. De igual manera, se puede resaltar la relación positiva entre todos los productos minero-energéticos. Hay que mencionar que la relación inversa más fuerte la comparte la tasa de cambio con los precios del petróleo, con un coeficiente mayor de -0,55. De igual manera, la correlación, entre los precios del petróleo y el carbón es alta, teniendo un coeficiente mayor a 0,7; es evidente que el comportamiento de los precios del petróleo Brent y W'TI es muy similar, con un coeficiente cercano a 1.

Figura 1. Comportamiento histórico de las variables usadas en el indice

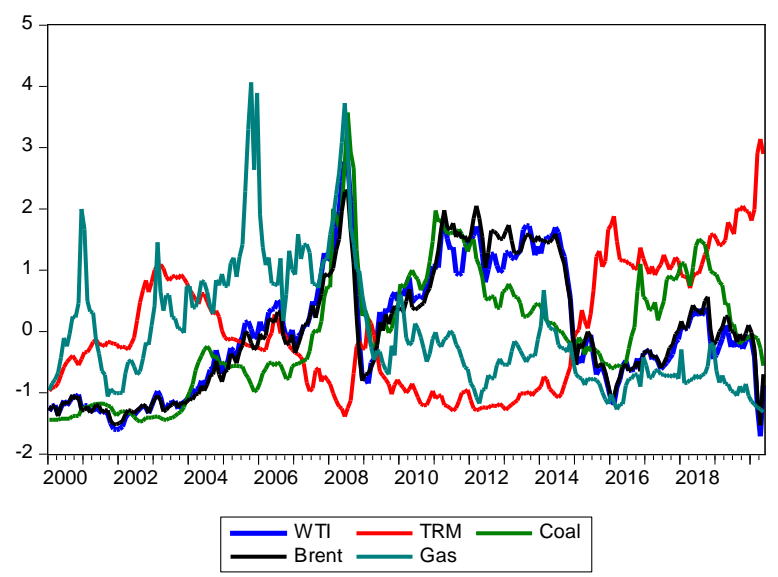

Fuente: elaboración propia.

Tabla 1. Coeficiente de correlación de series originales

\begin{tabular}{lccccc}
\hline & TRM & GAS & COAL & BRENT & WTI \\
\hline TRM & 1 & & & & \\
GAS & $-0,328$ & 1 & & & \\
COAL & $-0,255$ & 0,01599 & 1 & & \\
BRENT & $-0,564$ & 0,1066 & 0,7915 & 1 & \\
WTI & $-0,594$ & 0,2199 & 0,7831 & 0,982297 & 1 \\
\hline
\end{tabular}

Fuente: elaboración propia. 
Para estimar el índice de incertidumbre, se multiplica por (-1) el precio de cada materia prima, con el fin de que estos tengan comportamiento conjunto con la tasa de cambio y no generar sesgos a la hora de analizar la incertidumbre. En esa medida, el factor a estimar está compuesto por las siguientes variables:

$$
\boldsymbol{X}_{t}^{\prime}=[T R M, W T I, \text { Brent, Gas, COAL }]^{\prime},
$$

donde cada uno de los vectores de información de las materias primas, se multiplica por (-1).

\section{Discusión de resultados}

La Figura 2 muestra los resultados del Índice de incertidumbre (IMIFE) propuestos en este trabajo. Inicialmente, se puede identificar que los altos niveles de incertidumbre están asociados a bajos precios de las materias primas y altos valores de la tasa de cambio. De igual manera, los bajos niveles de incertidumbre están asociados a altas valoraciones de las materias primas y bajas cotizaciones de la tasa de cambio colombiana; lo cual concuerda con autores como Candelo (2018), Gómez (2015), González y Hernández (2016), Mendoza y Vera (2010), Oviedo y Candelo (2020) y Poncela et al. (2017), quienes afirman que la economía colombiana se ve afectada positivamente por los altos precios de las materias primas y que existe un comportamiento contrario respecto la tasa de cambio.

Respecto a los momentos de crisis como el 2008, se puede notar que este índice tiene un aumento vertiginoso, lo cual permite identificar que en la crisis subprime, la economía nacional pasó de bajos niveles de incertidumbre debido a los precios históricos de las materias primas, a altos momentos de incertidumbre cuando los mismos tienen una caída vertiginosa. En esa misma línea, a mitades del 2014 donde los precios de los hidrocarburos bajaron por factores ya mencionados, existe un aumento de este índice, e igualmente un menor crecimiento de la economía nacional. También este indicador muestra aumentos de la incertidumbre, en el año 2018 por la guerra comercial entre Estados unidos y China, y a su vez, muestra niveles de incertidumbre históricos en abril del 2020, debido a la llegada del COVID 19 a Colombia. 
Figura 2. Estimación del indice mensual de incertidumbre financiera y económica (IMIFE)

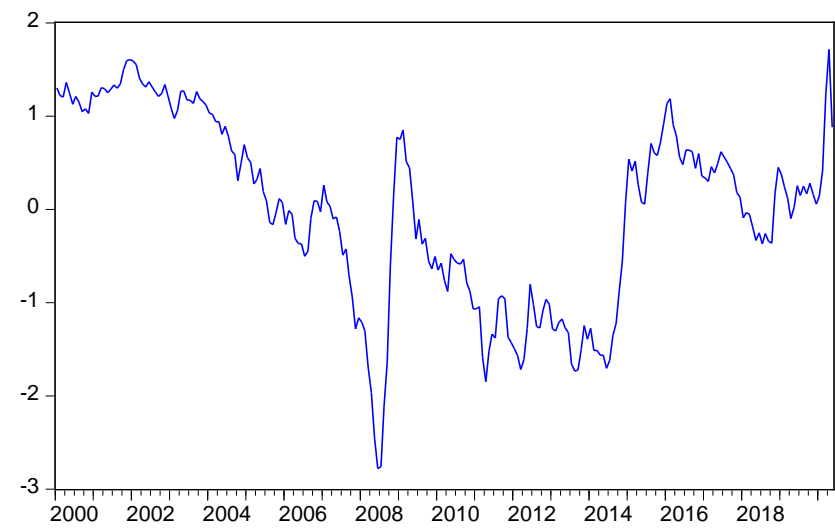

Fuente: elaboración propia.

De igual manera, vale resaltar que este índice también capta los pequeños momentos de incertidumbre en la economía, puesto que evidencia pequeños aumentos en momentos como lo fueron el 11 de septiembre del 2001 (en el atentado de las torres gemelas) y en el mes de octubre del 2016 (donde se efectuó el plebiscito por la paz en Colombia). es importante resaltar que, aunque este índice capta esos pequeños momentos de incertidumbre, sus mayores beneficios se notan en momentos de grandes crisis económicas.

La Figura 3 muestra la comparación del índice de incertidumbre calculado y el índice de precio de las acciones colombianas normalizados. Inicialmente, se puede observar el comportamiento contrario que tienen dichos indicadores, lo que permite identificar que los momentos donde aumenta la incertidumbre, los precios —en general- de las acciones colombianas disminuyen. Lo anterior, muestra la consistencia que tiene este indicador al momento de dar indicios de incertidumbre, puesto que autores como Asness et al. (2013) y Moskowintz et al. (2012) identifican que en los tiempos de incertidumbre el valor de las empresas en el tiempo cambia.

Para determinar el impacto que tiene la incertidumbre en la economía se ha planteado un vector autorregresivo (VAR) propuesto por Sims (1980), el cual resuelve el problema de la endogeneidad de las variables, permitiendo así 
observar el efecto de un choque de una variable sobre la otra. Para resolver la estimación de este modelo, se usa el indicador de seguimiento económico (ISE) para Colombia y el IMIFE. Se utilizan datos mensuales del año 2005 hasta mayo de 2020 por la disponibilidad de estos. Para la estimación de este modelo, se usan las variables en diferencias con el fin de analizar el ciclo económico. Vale mencionar que las series, en su primera diferencia son estacionarias, y el modelo se estima a 13 rezagos donde se cumple la no autocorrelación.

Figura 3. IMIFE vs indice de precios de las acciones colombianas

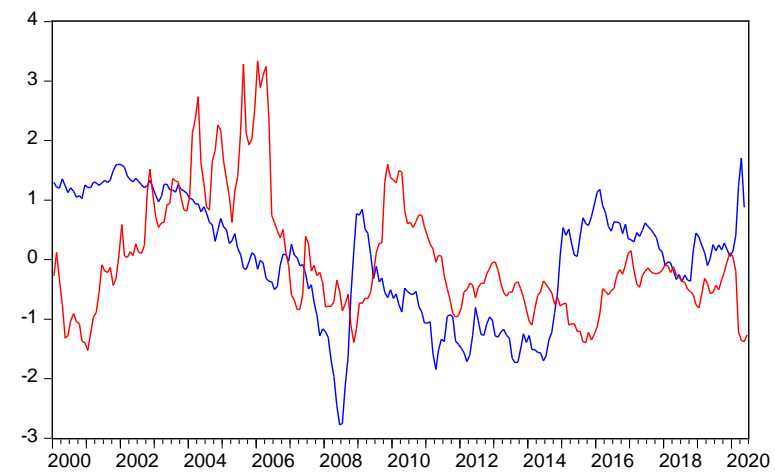

Fuente: elaboración propia.

La Figura 4 muestra la función impulso respuesta generalizada del modelo a dos desviaciones estándar, donde se puede identificar que la economía colombiana reacciona negativamente al aumento de la incertidumbre, mostrando así una relación negativa entre los momentos de alta incertidumbre y la actividad económica.

Dados los resultados del índice, este puede ser usado por diferentes agentes, como los inversionistas, a quienes les permite identificar caídas del mercado accionario. De igual forma puede ser usado por los elaboradores de política económica, en la medida que altos niveles de incertidumbre están asociados a un bajo crecimiento económico. Por último, este indicador también puede ser usado por los gerentes de las empresas privadas con el fin de redireccionar sus estrategias para el cumplimiento de los objetivos organizacionales. 
Figura 4. Función impulso respuesta generalizada del modelo a dos desviaciones estándar

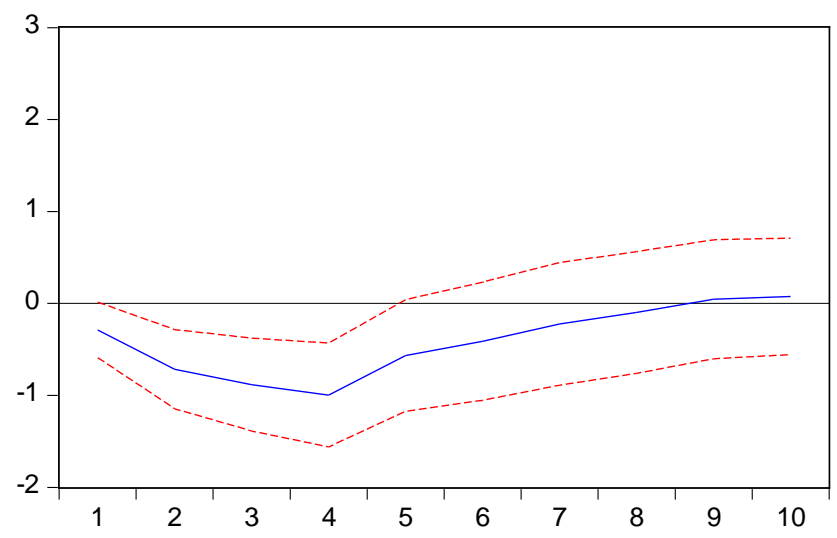

Nota: respuesta acumulada del ISE frente al IMIFE

Fuente: elaboración propia.

\section{Conclusiones}

Este artículo tuvo como objetivo desarrollar un índice que permita medir los niveles de incertidumbre en la economía colombiana. Para ello se siguió la metodología que permite la estimación de variables no observables, donde se aplican los modelos dinámicos factoriales propuestos por Geweke (1977) y - a su vez- lo aplica en una extensión del problema Stock y Watson (2002, 2005, 2011).

Entre los resultados —inicialmente- se puede identificar que los altos niveles de incertidumbre están asociados a bajos precios de las materias primas y altos valores de la tasa de cambio. De igual manera, los bajos niveles de incertidumbre están asociados a altas valoraciones de las materias primas y bajas cotizaciones de la tasa de cambio colombiana. Igualmente, en los momentos de crisis — como el caso del año 2008 - , se puede notar que este índice tiene un aumento acelerado, lo cual permite identificar que en la crisis subprime la economía nacional pasó de bajos niveles de incertidumbre - por precios históricos de las materias primas- a altos momentos de incertidumbre cuando los mismos tiene una caída vertiginosa. También se 
puede notar que este índice tiene un aumento acelerado, lo cual permite identificar que en la crisis subprime la economía nacional pasó de bajos niveles de incertidumbre — debido a los precios históricos de las materias primas - a altos momentos de incertidumbre cuando los mismos tienen una caída vertiginosa. En esa misma línea, a mitades del año 2014 —cuando los precios de los hidrocarburos bajaron por factores ya mencionados-, existe un aumento de este índice, e igualmente un menor crecimiento de la economía nacional. Este indicador también muestra aumentos de la incertidumbre en el año 2018 por la guerra comercial entre Estados unidos y China y, a su vez, muestra niveles de incertidumbre históricos en abril del 2020 debido a la llegada del COVID 19 a Colombia.

Por último, se realizó la comparación del IMIFE calculado y el índice de precios de las acciones colombianas. Inicialmente, se puede observar el comportamiento contrario que tienen dichos indicadores, lo que permite inferir que los momentos donde aumenta la incertidumbre, los precios de las acciones colombianas disminuyen. Lo anterior, muestra la consistencia que tiene este indicador al momento de dar indicios de incertidumbre. En esa misma línea, se puede probar que la economía colombiana reacciona negativamente al aumento de la incertidumbre, mostrando así una relación negativa entre los momentos de alta incertidumbre y la actividad económica.

\section{Agradecimientos}

Este artículo se deriva de la tesis del autor para optar al título de doctor en administración en la línea de finanzas cuantitativas de la Universidad del Valle, Cali-Colombia.

\section{Referencias}

Ali, U., Daniel, K. D., \& Hirshleifer, D. A. (2017). One briefshining moment (um): Past momentum performance and momentum reversals [Columbia Business School Research Paper, 17-48]. https://papers.ssrn.com/sol3 /papers.cfm?abstract_id=2956493 
Candelo-Viáfara: Índice mensual de incertidumbre financiera y económica (IMIFE)...

Asness, C. S., Moskowitz, T. J., \& Pedersen, L. H. (2013). Value and momentum everywhere. The Journal of Finance, 68(3), 929-985. https: //doi.org/10.1111/jofi.12021

Baker, S. R., Bloom, N. \& Davis, S. J. (2016). Measuring Economic Policy Uncertainty. The Quarterly Journal of Economics, 131(4), 1593-1636. https://doi.org/10.1093/qje/qjw024

Bernanke, B. S. (1983). Irreversibility, Uncertainty and Cyclical Investment. Quarterly Journal of Economics, 98(1), 85-106. https://doi.org/10.230 $7 / 1885568$

Bernstein, P. L. (1998). Against the Gods: the remarkable story of Risk. John Wiley \& Sons. Inc.

Basher, S. A., \& Sadorsky, P. (2016). Hedging emerging market stock prices with oil, gold, VIX, and bonds: A comparison between DCC, ADCC and GO-GARCH. Energy Economics, 54, 235-247. https://doi.org/10 $.1016 /$ j.eneco.2015.11.022

Birchenall, J. (1997). Inversión, q de Tobin, e incertidumbre en la industria colombiana. Revista Desarrollo y Sociedad, 39, 149-207. https://doi.or g/10.13043/dys.39.6

Brander, J., \& Krugman, P. (1983). A reciprocal dumping'model of international trade. Journal of International Economics, 15(3-4), 313321. https://doi.org/10.1016/S0022-1996(83)80008-7

Bloom, N. (2009). The impact of uncertainty shocks. Econometrica, 77(3), 623-685. https://doi.org/10.3982/ECTA6248

Brahmasrene, T., Huang, J. C., y Sissoko, Y. (2014). Crude oil prices and exchange rates: Causality, variance decomposition and impulse response. Energy Economics, 44, 407-412. https://doi.org/10.1016/j. eneco.2014.05.011

Candelo, J. M. (2018). Impactos indirectos de la tasa de cambio y los precios del petróleo en una economía no petrolera: aproximaciones VECM y VAR para el Valle del Cauca, Colombia. Finanzas y Política Económica, 
10(2), 403-436. http://dx.doi.org/10.14718/revfinanzpolitecon.2018. 10.2.9

Cerda, R., Silva, Á. \& Valente, J. (2016). Economic Uncertainty Impact in a Small Open Economy: The Case of Chile. Centro Latinoamericano de Politicas Económicas y Sociales [document de trabajo]. Universidad del Desarrollo. https://negocios.udd.cl/files/2016/12/CerdaSilvaValent e_EU_Chile_Paper.pdf

Cooper, M. J., Gutierrez Jr, R. C., \& Hameed, A. (2004). Market states and momentum. The journal of Finance, 59(3), 1345-1365. https: //doi.org/10.1111/j.1540-6261.2004.00665.x

Daniel, K., Hirshleifer, D., \& Subrahmanyam, A. (1998). Investor psychology and security market under-and overreactions. the Journal of Finance, 53(6), 1839-1885. https://doi.org/10.1111/0022-1082.0007 7

Daniel, K., \& Moskowitz, T. J. (2016). Momentum crashes. Journal of Financial Economics, 122(2), 221-247._https://doi.org/10.1016/j.jf ineco.2015.12.002

de Truchis, G., \& Keddad, B. (2016). On the risk comovements between the crude oil market and US dollar exchange rates. Economic Modelling, 52(Part A), 206-215. https://doi.org/10.1016/j.econmod.2014.11.014

Delgado-Rojas, M. \& Rincón-Castro, H. (2017). Incertidumbre acerca de la política fiscal y ciclo económico [documento de trabajo]. Borradores de economía 1008. Banco de la República. http://repositorio.banrep.gov .co/bitstream $/$ handle/20.500.12134/6321/be_1008.pdf?sequence $=5$

Frazzini, A. (2006). The disposition effect and underreaction to news. The Journal of Finance, 61(4), 2017-2046. https://doi.org/10.1111/j.1540 $-6261.2006 .00896 . x$

FRED Economic Data. (2020). Economic Research. Federal Reserve Bank of St. Louis. https://fred.stlouisfed.org/ 
Candelo-Viáfara: Índice mensual de incertidumbre financiera y económica (IMIFE)...

Gervais, S., Kaniel, R., \& Mingelgrin, D. H. (2001). The high-volume return premium. The Journal of Finance, 56(3), 877-919. https://doi.org/10.1 111/0022-1082.00349

Geweke, J. (1977). The dynamic factor analysis of economic time series. En D. J. Aigner \& A. S. Goldberger (eds.): Latent Variables in Socioeconomic Models (pp. 365-383). North-Holland Publications.

Gil-León, J. M. \& Silva-Pinzón, D. E. (2019). Índice de incertidumbre de política económica (EPU) para Colombia, 2000-2017. Ensayos de Economí, 29(55), 37-56. https://doi.org/10.15446/ede.v29n55.7622 4

Golub, S. S. (1983). Oil prices and exchange rates. The Economic Journal, 93(371), 576-593. https://doi.org/10.2307/2232396

Gómez, C. (2015). Más allá de un boom de recursos naturales: efectos de los choques petroleros en la economía colombiana [Documentos CEDE012565]. Universidad de Los Andes. https://ideas.repec.org/ $\mathrm{p} / \mathrm{col} / 000089 / 012565 . \mathrm{html}$

González, S., \& Hernández, E. (2016). Impactos indirectos de los precios del petróleo en el crecimiento económico colombiano. Lecturas de Economia, (84), 103-141. https://doi.org/10.17533/udea.le.n84a04

Haddow, A., Hare, C., Hooley, J., \& Shakir, T. (2013). Macroeconomic Uncertainty: What Is It, How Can We Measure It and Why Does It Matter? [Bank of England Quaterly Bulletin Q2]. https://www.bankofengland. co.uk/-/media/boe/files/quarterly-bulletin/2013/macroeconomic-un certainty-what-is-it-how-can-we-measure-it-and-why-does-it-matter. pdf?la=en\&hash=A607045F0DA716475FD8CCC9A2313F019394 A264

Hong, H., \& Stein, J. C. (1999). A unified theory of underreaction, momentum trading, and overreaction in asset markets. The Journal of Ffinance, 54(6), 2143-2184. https://doi.org/10.1111/0022-1082.00 184 
Johnson, T. C. (2002). Rational momentum effects. The Journal of Finance, 57(2), 585-608. https://doi.org/10.1111/1540-6261.00435

Keynes, J. M. (1921). A treatise on probability. Macmillan and Company, limited.

Keynes, J. M. (1939). The League of Nations Professor Tinbergen's Method. The Economic Journal, 49(195), 558-577. https://doi.org/10.1093/ej/4 9.195 .558

Knight, F. H. (1921). Risk, Uncertainty, and Profit. Library of Economics and Liberty. http://www.econlib.org/library/Knight/knRUP1.htm

Krugman, P. (1983). Oil shocks and exchange rate dynamics. En J. A. Frenkel (ed). Exchange rates and international macroeconomics (pp. 259-284). University of Chicago Press. http://www.nber.org/chapters/c11382

Liu, L. X., \& Zhang, L. (2008). Momentum profits, factor pricing, and macroeconomic risk. The Review of Financial Studies, 21(6), $2417-$ 2448. https://doi.org/10.1093/rfs/hhn090

Lof, M., \& Nyberg, H. (2017). Noncausality and the commodity currency hypothesis. Energy Economics, 65, 424-433. https://doi.org/10.1016/j. eneco.2017.05.024

Mendoza, O. \& Vera, D. (2010). The asymmetric effects of oil shocks on an oil-exporting Economy. Cuadernos de Economía, 47(135), 3-13. http: //dx.doi.org/10.4067/S0717-68212010000100001

Moskowitz, T. J., Ooi, Y. H., \& Pedersen, L. H. (2012). Time series momentum. Journal of Financial Economics, 104(2), 228-250. https: //doi.org/10.1016/j.jfineco.2011.11.003

Novy-Marx, R. (2012). Is momentum really momentum? Journal of Financial Economics, 103(3), 429-453. https://doi.org/10.1016/j.jfineco.2011.0 5.003

Ortiz, C. H. (2016). Diversificación productiva y crecimiento económico. Programa Editorial, Universidad del Valle. 
Candelo-Viáfara: Índice mensual de incertidumbre financiera y económica (IMIFE)...

Oviedo, A. F., \& Sierra, L. P. (2019). Importancia de los términos de intercambio en la economía colombiana. Revista de la CEPAL, (128), 125-154. https:/www.cepal.org/sites/default/files/publication/files/44 740/RVE128_Oviedo.pdf

Oviedo-Gómez A. F. \& Candelo-Viafara, J. M. (2020). Mining and energy commodity price effects on colombian economic. Cuadernos de Administración, 36 (67), 93-108. https://cuadernosdeadministracion. univalle.edu.co/index.php/cuadernos_de_administracion/article/view /8641

Partow, Z. (1996). Incertidumbre económica e inversión privada en Colombia [documento de trabajo]. Borradores de Economía, 56. Banco de la República. http://www.banrep.gov.co/sites/default/files/publicacio nes/pdfs/borra056.pdf

Poncela, P., Senra, E., \& Sierra, L. P. (2017). Long-term links between raw materials prices, real exchange rate and relative de-industrialization in a commodity-dependent economy: empirical evidence of "Dutch disease" in Colombia. Empirical Economics, 52(2), 777-798. https://doi.org/10 .1007/s00181-016-1083-7

Posada, H. M. (2010). Incertidumbre macroeconómica e inversión real en Colombia. Sociedad y Economía, 18, 269-300. https://core.ac.uk/dow nload/pdf/11860684.pdf

Sims. C. A. (1980) Macroeconomics and Reality. Econometrica, 48(1), 1-48. https://doi.org/10.2307/1912017

Stock, J. H., \& Watson, M. W. (2002). Macroeconomic forecasting using diffusion indexes. Journal of Business \& Economic Statistics, 20(2), 147-162. https://doi.org/10.1198/073500102317351921

Stock, J. H., \& Watson, M. W. (2005). Implications of dynamic factor models for VAR analysis [working paper NBE 11467]. National Bureau of Economic Research, 1-67. https://papers.ssrn.com/sol3/papers.cf m?abstract_id $=755703$ 
Stock, J. H., \& Watson, M. W. (2011). Dynamic factor models. Oxford handbook of Economic for Recasting, 1, 35-59. https://doi.org/10.1093/ oxfordhb/9780195398649.013.0003

Streb, J. M. (2000). Por qué importan las instituciones políticas para el desempeño económico: incertidumbre politica y subdesarrollo [documento de trabajo]. Universidad del CEMA. https://ucema.edu.ar/publicaciones/downloa d/documentos/168.pdf

World Bank. (2015). Global Economic Prospects, June 2015: The Global Economy in Transition. The World Bank. https://www.worldbank. org/content/dam/Worldbank/GEP/GEP2015b/Global-Economic-P rospects-June-2015-Global-economy-in-transition.pdf 